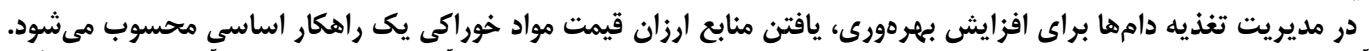

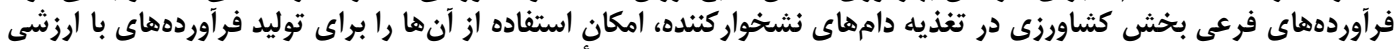

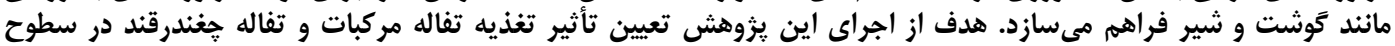

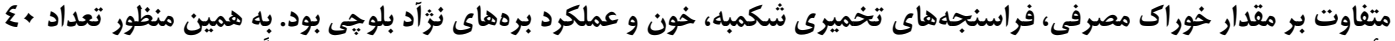

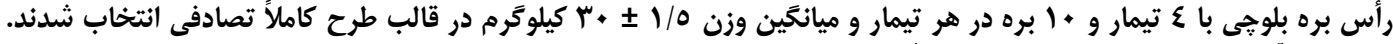

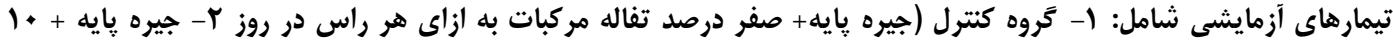

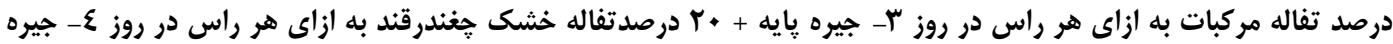

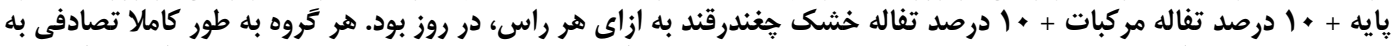

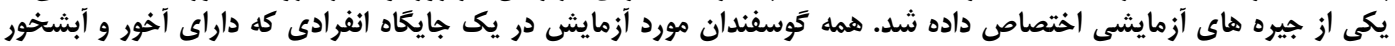

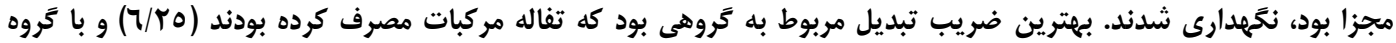

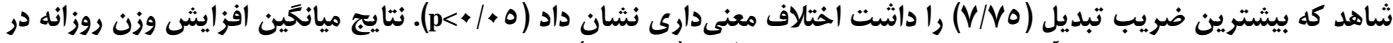

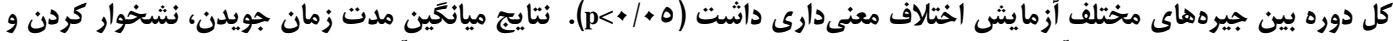

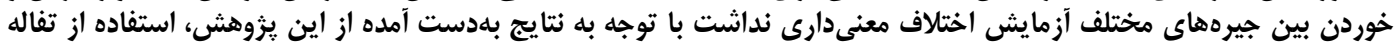

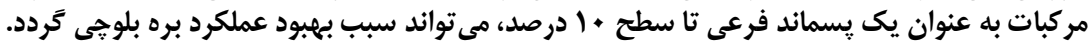

كسترش در تنوع محصولات و نيز ييشرفت صنايع غذايي،

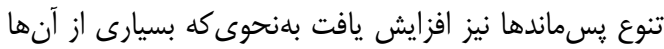

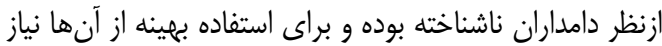

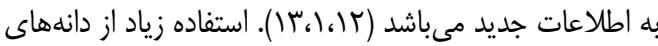

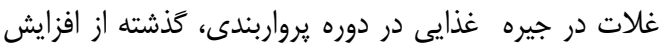

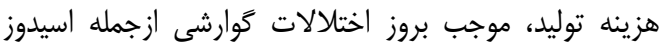

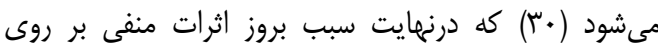

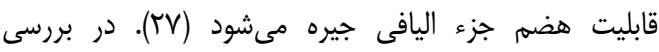

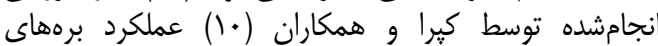

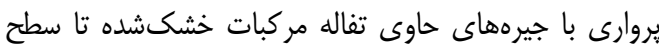

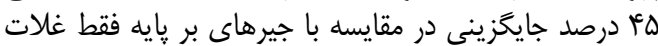

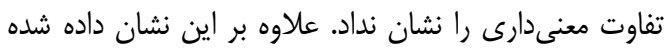

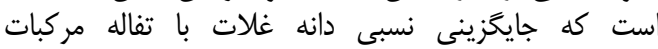

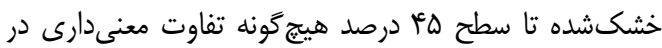

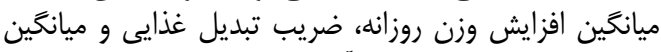

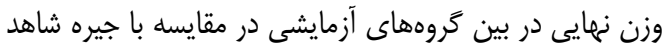

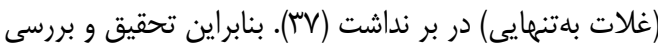

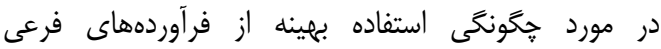

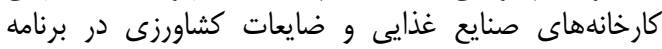

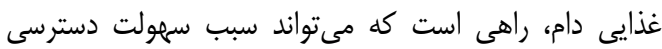

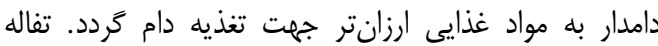

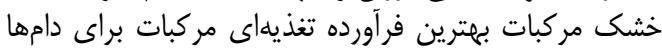

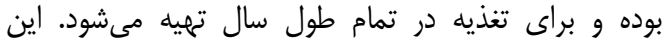

وازههاى كليدى: يسماند مركبات، نشخوار، تَوسفند، جغندرقند

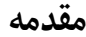

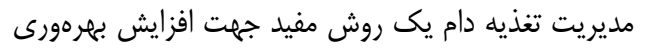

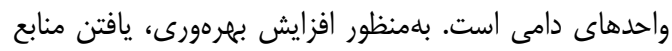

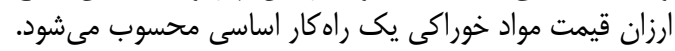

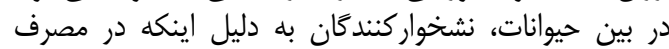

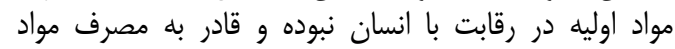

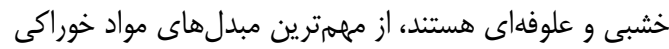

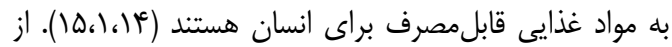

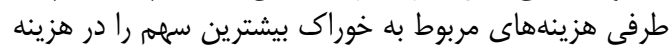

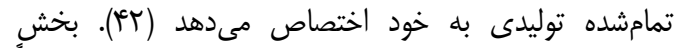

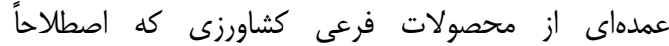

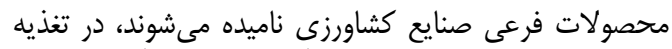

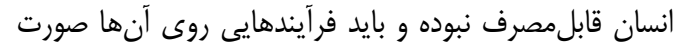

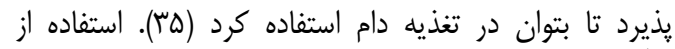

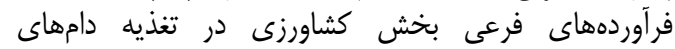

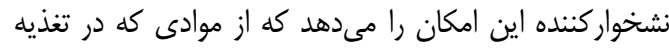

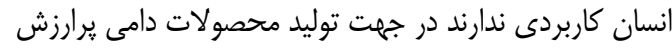

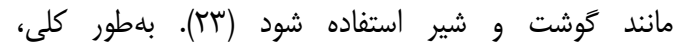
يسماندهاى كشاورزى و منابع طبيعى شامل موادى هستند كه إنه

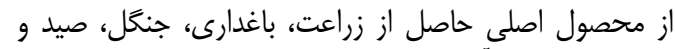

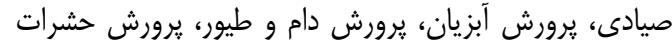

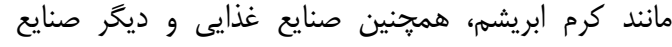

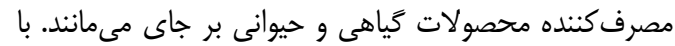




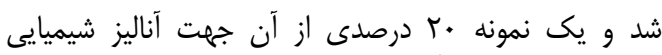

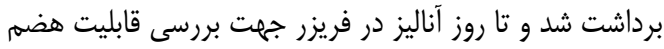

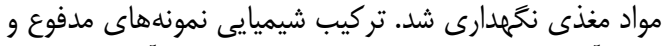

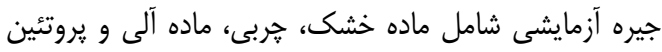

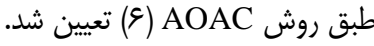

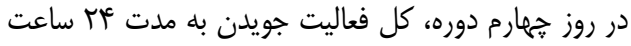

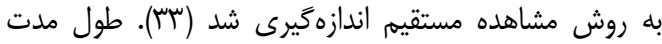

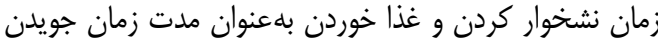

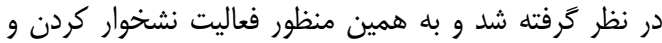

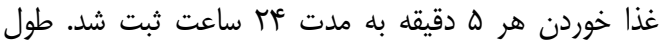

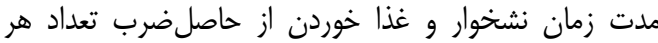

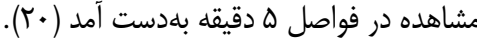

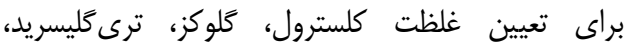

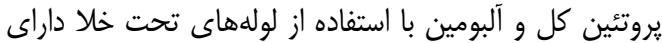

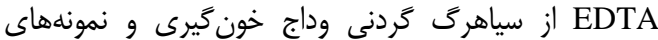

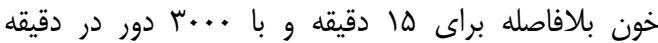

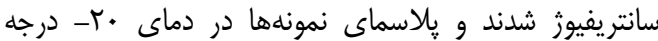

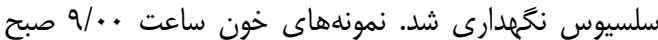

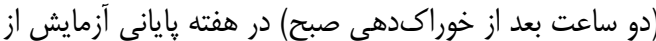

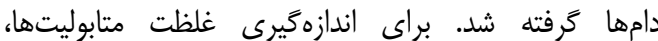

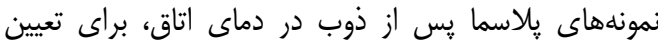

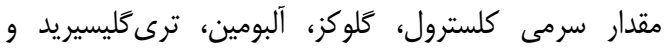

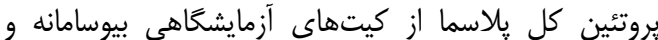
دستخاه اتوآنالايزر (مدل A15 فرانسه) با دو تكرار اندازهكيرى

\section{تجزيه و تحليل آمارى}

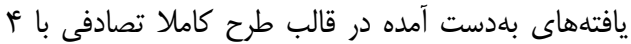
تيمار و 9 تكرار در هر تيمار بود و به شرح مدل زير ترام تجزيه تاديه

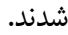

$Y i j=\mu+T i+\varepsilon i j$

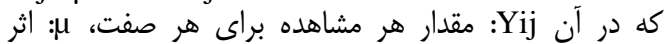

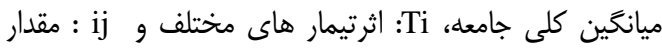
خطاى باقيمانده بود.

تحليل دادههاى نظير مصرف خوراك، وزن بدن، نجان نرمافزار

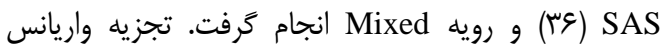

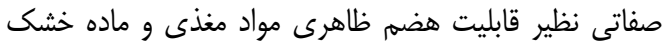

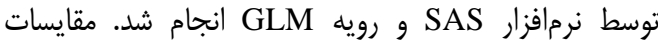

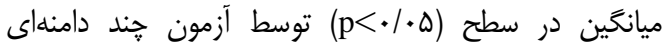

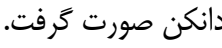

محصول به سهولت انبارشده و حملونقل آن آسان است.

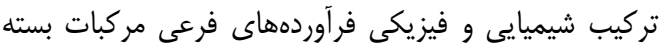

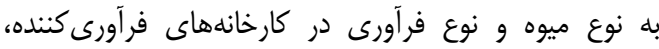

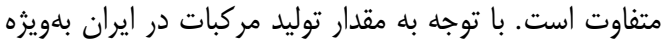

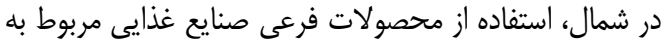

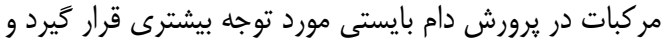

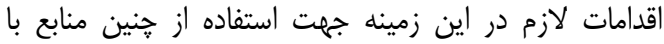
ارزشى صورت كيرد. اين آزمايش با هدف تعيين تأثير تغذيه تفاله مركبات و ورئ

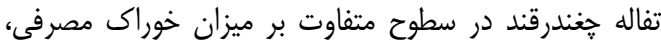

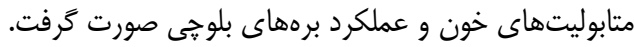

\section{مواد و روشها}

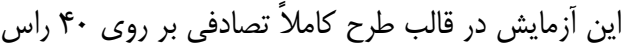

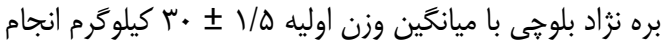

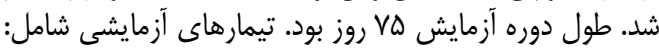

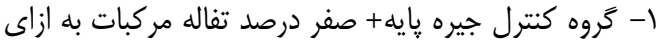

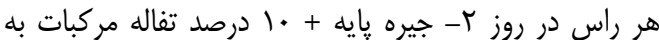

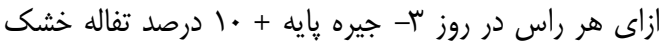

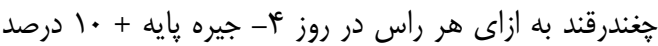

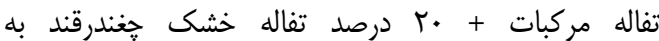

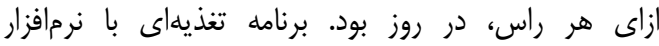
Small Ruminant Nutrition System (SRNS)

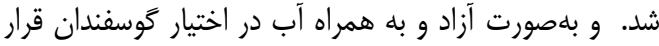

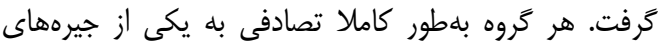

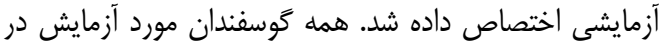

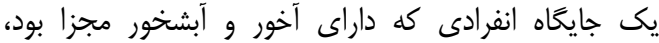
نَخهدارى شدند.

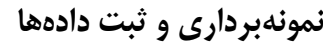

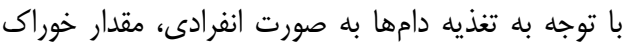

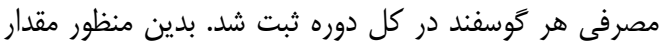

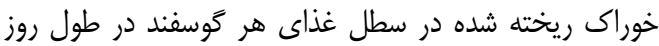

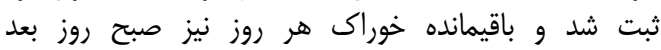

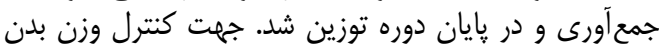

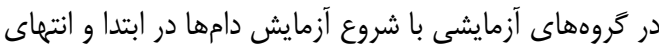

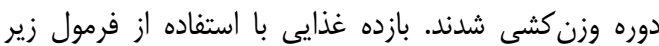

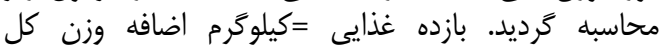

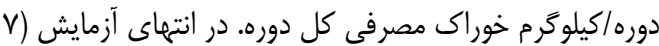

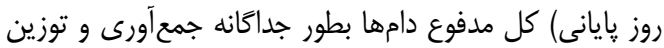


Table 1. The composition and chemical composition of the diet on Baluchi lambs

جدول ا- اجزاى مواد خوراكى جيرههاى آزمايشى (بر اساس درصد ماده خشك) برههاى بلوجى

\begin{tabular}{|c|c|c|c|c|}
\hline \multicolumn{5}{|c|}{ تيمار" } \\
\hline تيمار f & تيمار r & تيمار r & تيمار ا(شاهد) & ماده خوراكى \\
\hline $19 / 4 r$ & $19 / 4 \mathrm{~V}$ & $19 / 4 \mathrm{~V}$ & $19 / 4 V$ & يونجه خشك \\
\hline 1. & . & 1. & . & تفاله مركبات \\
\hline 1. & r. & . & . & تفاله خشك حتندرقند \\
\hline V/Ar & V/Ar & $11 / \lambda r$ & W/AT & سيلاز ذرت \\
\hline IF/AT & $\mid F / A r$ & IF/Ar & IF/Ar & كاه جو \\
\hline $1 . / \% \Delta$ & $1 . / \% \Delta$ & $\mid \pi / \pi \Delta$ & $\mid \omega / \sim \Delta$ & دانه جو \\
\hline$\Delta / \sim \Delta$ & $\Delta / \Gamma^{\prime} \Delta$ & $g / \pi \Delta$ & $\mathrm{v} / \mathrm{r \omega}$ & دانه ذرت \\
\hline$\Delta / r T$ & D/Tr & g/r & s/rT & كنجاله سويا \\
\hline $1 / 8$. & $1 / 8$. & $1 / 9$. & $1 / 9$. & يودر هربى \\
\hline$N / 9 \Delta$ & $N / 9 \Delta$ & N/9D & N/9D & سبوس \\
\hline$\cdot|\wedge|$ & $\cdot|\Lambda|$ & $\cdot|\wedge|$ & $\cdot|\wedge|$ & كربنات كلسيم \\
\hline$\Delta / \bar{\phi}$. & Q/द. & s/s. & s/s. & كنجاله تخم ينبه \\
\hline
\end{tabular}

جدول r- تركيب شيميايى (درصد در ماده خشك) و انرزى (مكا كالرى در كيلوكرم) Table 2. The chemical composition (\% of dry) and energy (megacal/kg) of the diet

\begin{tabular}{|c|c|}
\hline 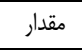 & تركيب شيميايى \\
\hline $\mid r / M$ & يروتئين خام (درصد) \\
\hline$r / \Lambda \mathcal{E}$ & انرزى قابل متابوليسم (مكا كالرى در كيلوكَرم) \\
\hline$r / M r$ & ل تهربى خام (درصد) \\
\hline$v \cdot / v$ & ماده خشى (درصد) \\
\hline$f \cdot / r f$ & الياف نامحلول در شوينده خنثى (درصد) \\
\hline ME/Tg & كربوهيدرات غير اليافى (درصد) \\
\hline$\cdot / M$ & كلسيم (درصد) \\
\hline.$/ 41$ & فسفر (درصد) \\
\hline
\end{tabular}

مشاهده نكردند. ولانيس و همكاران (qس) دريافتند كه سيلاز

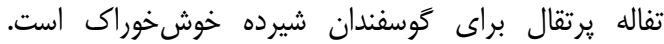

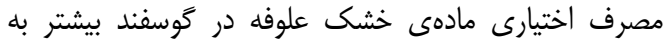

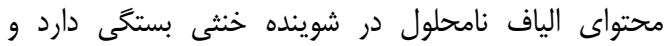

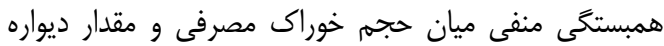

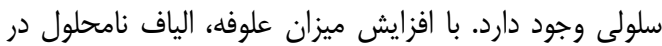

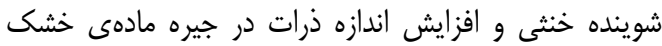

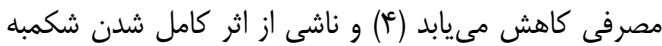

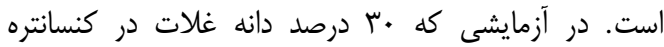

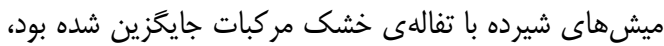

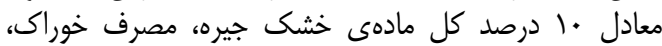

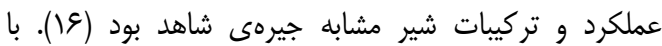

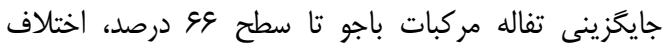

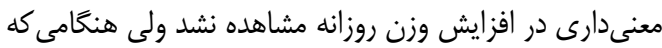

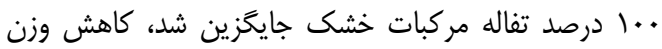

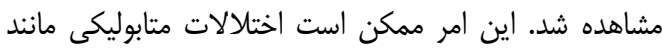

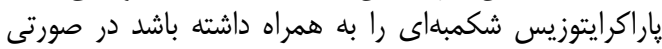

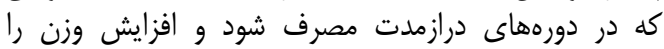

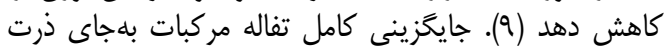

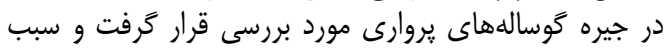

نتايج و بحث

عملكرد

نتايج مربوط به عملكرد (ميانخين خوراك مصرفى، ضريب

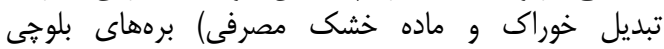

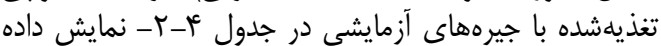

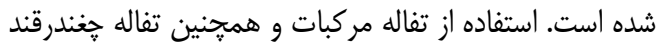

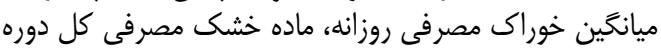

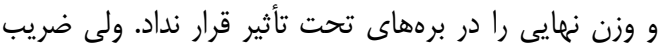

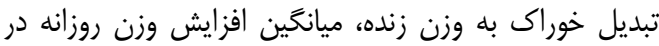

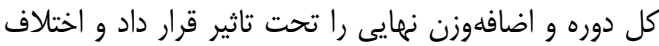

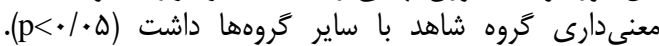

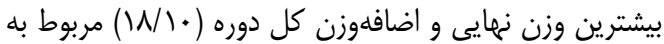

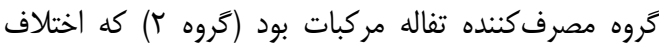

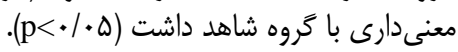

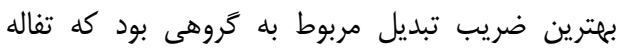

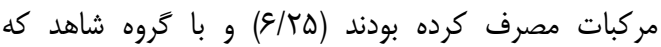

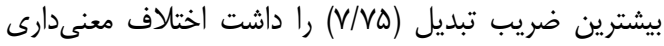

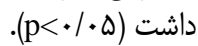

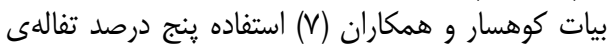

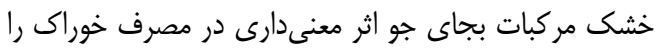


دانه تقطيرى خشكشدهى جو، ضريب تبديل غذايى را بلهطور

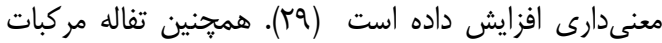

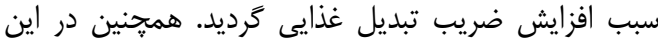

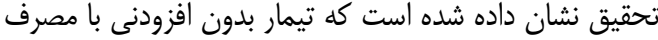

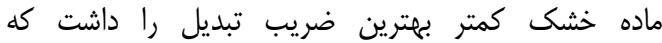
كشاندهندهى راندمان بهتر استفاده از مواد مغذى جير جيره است كه مغاير با نتايج اين تحقيق بود.

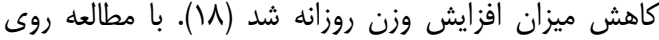

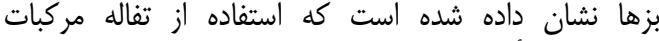

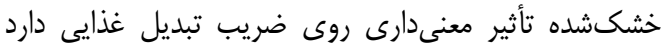

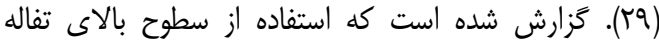

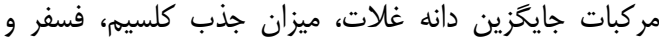

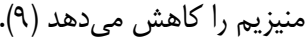
كزارش شده است VD درصد جايكزينى تفالهى مركبات با

جدول بـ- تاثير جيرههاى آزمايشى بر ميانگين خوراك مصرفى، ضريب تبديل غذايى برههاى بلوجى Table 3. Effect of experimental diets on functional parameters of Baluchi lambs

\begin{tabular}{|c|c|c|c|c|c|c|}
\hline \multirow[b]{2}{*}{ سطح احتمال } & \multirow[b]{2}{*}{ خطاى استاندارد ميانگَين } & \multicolumn{4}{|c|}{ 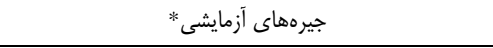 } & \multirow[b]{2}{*}{ فراسنجهها } \\
\hline & & 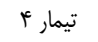 & تيمار & ت تيمار r & تيمار 1 & \\
\hline.$/ 1 f r$ & 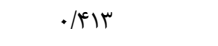 & $1 / \Delta \Delta$ & $1 / \Delta$ & $1 / 01$ & $1 / 94$ & خوراك مصرفى روزانه (كيلوگرم در روز) \\
\hline$\cdot / T / \Lambda \Delta$ & $F / \Delta \cdot 1$ & $11 \varepsilon / r \Delta$ & $\| F / V \Delta$ & $11 \% / r \Delta$ & $|r| / \Delta \mid$ & خوراك مصرفى كل دوره (كيلوگرم) \\
\hline ./vas. & $r / 9 \cdot r$ & $r \Delta / \& q$ & 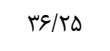 & $r V / \perp Q$ & $r \Delta / \Delta)$ & وزن اوليه (كيلوگرم در روز) \\
\hline$\cdot 1 \cdot 9 \cdot 1$ & T/TIQ & $\Delta T / Q G$ & $\Delta r / 9 \Delta$ & $\Delta \omega / T \Delta$ & $\Delta \cdot / 9 \Delta$ & وزن نهايى (كيلوگرم در روز) \\
\hline $.1 \cdots+1$ & Tr/VG. & $r r \cdot / v \Delta^{a}$ & 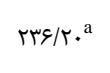 & $r f / / 0 \cdot{ }^{a}$ & $r \cdot \Delta / ৭ \Delta^{b}$ & در ريانكَين افزايش وزن روزانه در كل دوره (گرم \\
\hline $.1 \cdots+1$ & 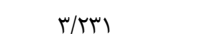 & $\mathrm{IV} / \mathrm{r} \cdot \cdot^{\mathrm{a}}$ & $\mathrm{IV} / \mathrm{V} \cdot{ }^{\mathrm{a}}$ & $M / *^{a}$ & $\mid Q / f e^{b}$ & اضافه وزن كل دوره (كيلوَرم) \\
\hline$\cdot|\cdot r|$ &.$/ 901$ & $g / v^{b}$ & $q / \uparrow \Lambda^{b}$ & $q / T \Delta^{b}$ & $V / V \Delta^{b}$ & ضريب تبديل خوراك به وزن زنده (كيلوگرم) \\
\hline 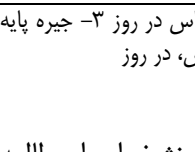 & 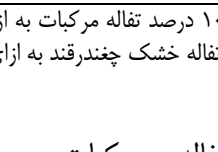 & 年, & 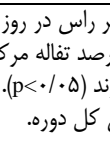 & 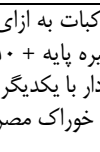 & درصد تفا در روز & 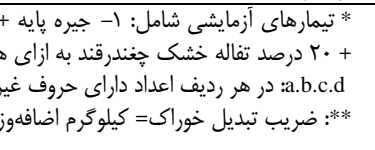 \\
\hline
\end{tabular}

و همكاران اثر تفالهى مركبات روى فعاليت نشخوار را مطالعه

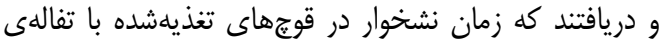

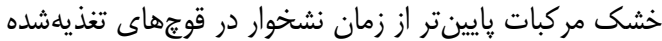

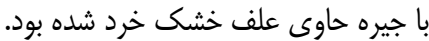

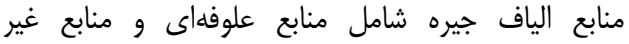

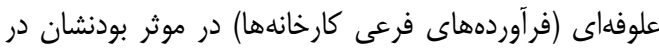

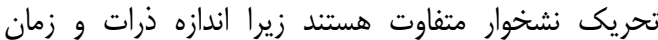

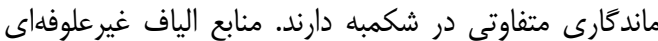

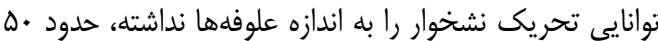

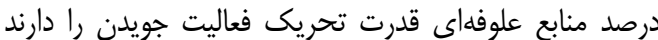

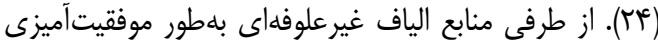

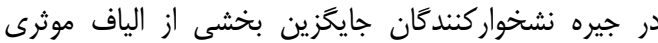

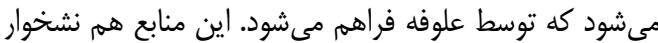

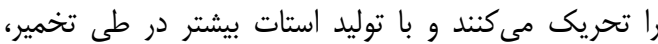

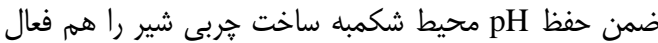

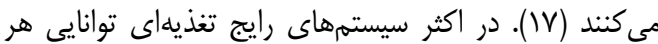

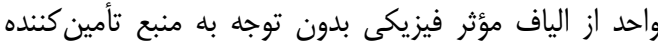
علوفه، مواد دانهاى يا فرآوردههاي فرئ فرعى در تحريك فئ فعاليت

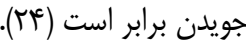

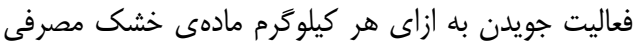

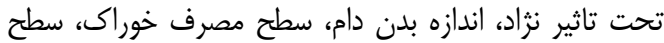

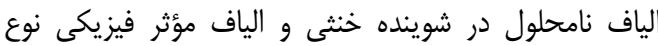

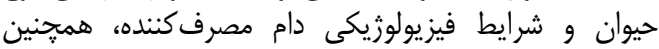

محتواى و ماهيت الياف جيره قرار مى گيرد (أم) دان.

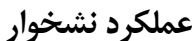
نتايج آمارى دادههاى حاصل إز از اين آزمايش نشان داد داد كه

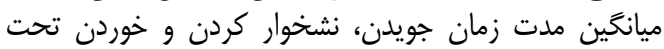

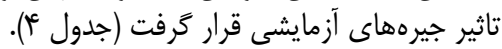

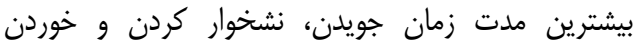

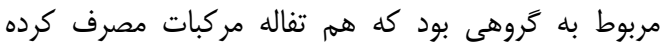

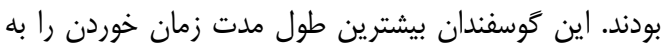

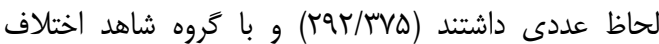

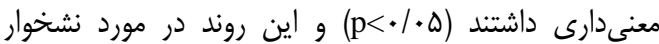

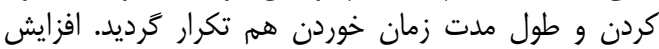

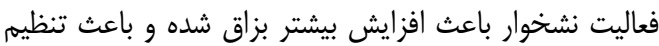

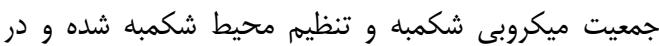

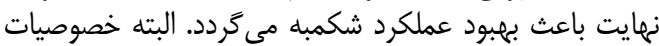

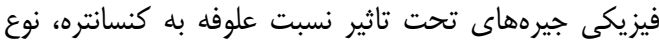

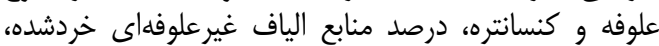

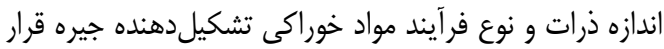

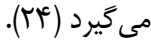
به دليل وجود مكانيسمهاى هومئوستاز و كنترل شديد توسط سيستم اعصاب و غداند، تغيير عوامل متابوليكانيك خون

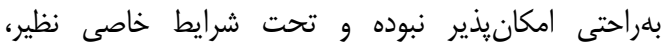

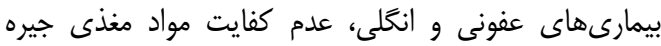

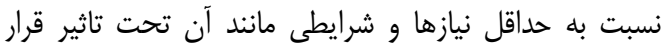

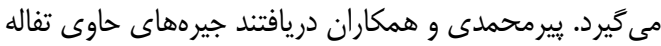

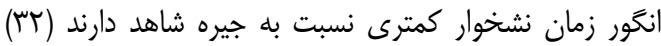

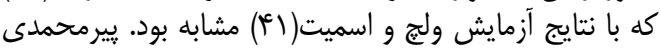


زمان صرف شده جهت نشخوار و كاهش زمان ماندگارى

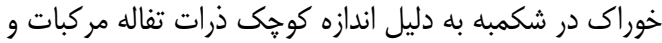

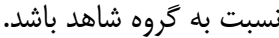

درمجموع مىتوان خنين نتيجه گيرى كرد كه يكى از عوامل

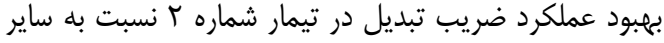

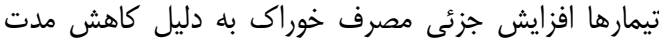

Table 4. Effect of experimental diets on eating time of Baluchi lambs

جدول ع- اثر جيرههاى آزمايشى بر مدت زمان جويدن در برهاى بلوجى

\begin{tabular}{|c|c|c|c|c|c|c|}
\hline \multicolumn{6}{|c|}{ جيرههاى آزمايشى* } & \multirow[b]{2}{*}{ شاخصهاى اندازمخيرى شده } \\
\hline سطح احتمال & خطاى استانداردميانكين & تيمار r & تيمار بr & تيمار r & تيمار I & \\
\hline$\cdot \cdot \cdot r \mid$ & אואוN & $M M / r \Delta T^{b}$ & $r \Lambda 1 / r F \Delta^{a}$ & $r q T / r \vee \Delta^{a}$ & $r \Lambda \cdot / \Lambda \vee \Delta^{\mathrm{a}}$ & 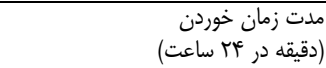 \\
\hline $.1 .+1 \Delta$ & $\mid T / 1 F \Delta$ & $F \mid r / r r \Delta^{b}$ & $\mu q . / r \mid \Delta^{b}$ & $r q \Lambda / r \Delta)^{b}$ & $\uparrow \Delta \Delta / \wedge r \Delta^{\mathrm{a}}$ & 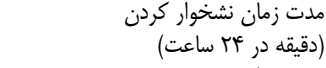 \\
\hline $.1 .+91$ & $\|F / V\|$ & $q \cdot \cdot / \leftarrow \wedge v^{b}$ & $s \mathrm{~V} / \Delta \mathrm{g}^{\mathrm{a}}$ & $99 . / 9 r q^{\mathrm{a}}$ & $v^{w} /(v) \cdot{ }^{a}$ & 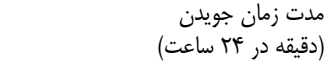 \\
\hline$\cdot / \cdot 1 \Delta 9$ & $N / \cdot V$ & $r . / r \& \Delta$ & TrV/QT. & TMI/Tr. & TTD/VTD & 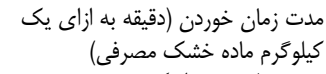 \\
\hline$\cdot / 1 \cdot r)$ & Q/Ar. & $T Y \Delta / \Delta T I$ & $r \Delta S / T \Delta \mid$ & $r \Lambda F / .1$. & $|F| r / \% A \mid$ & مصرفى) إزاى يك كيلوكرم ماده خشى \\
\hline . $\mid$ IMTI & $|0 / 1 \Lambda|$ & $\Delta f \Delta / V N S$ & $\Delta \Lambda \mu / W(W$ & $s / \Delta / r \Delta$. & $9^{\mu} N / 1.9$ & 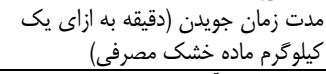 \\
\hline
\end{tabular}

نشاندهنده بروز اختلال در روند متابوليسم كربوهيدراتها

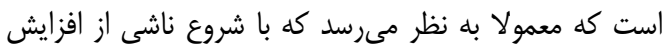

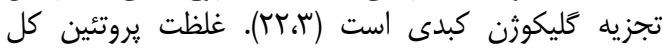

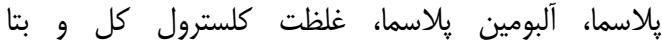

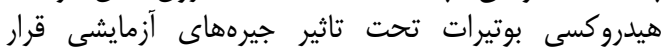

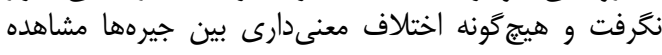

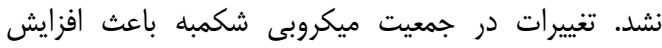

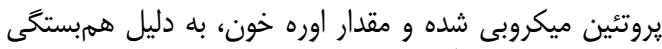

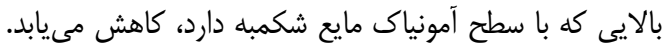

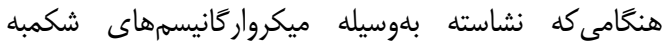

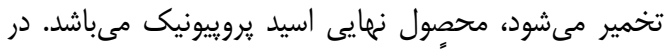

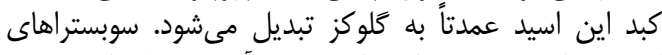

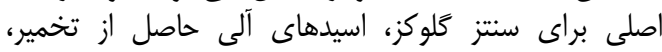

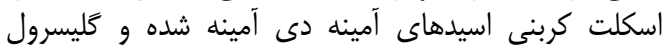

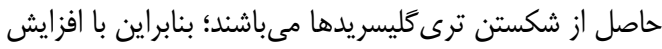

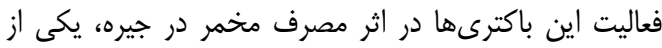

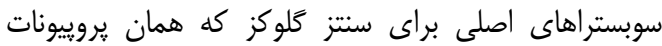

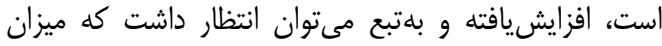

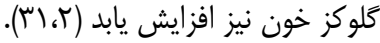

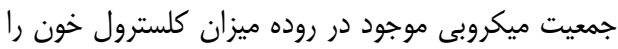

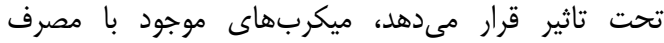

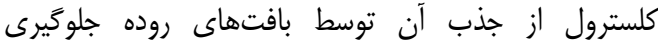

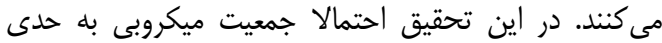

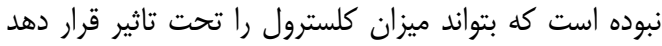

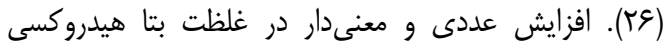

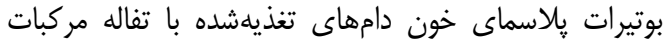

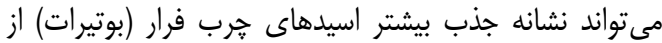

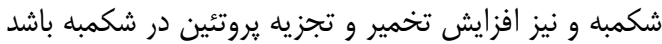

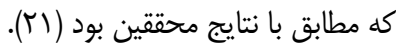

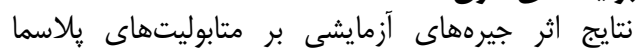

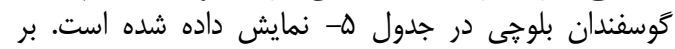

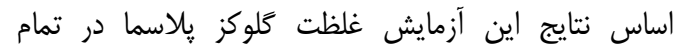

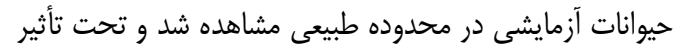

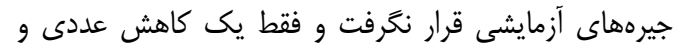

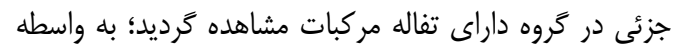

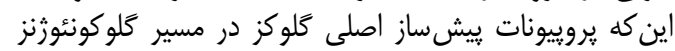

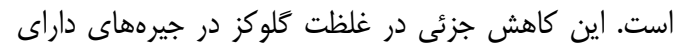

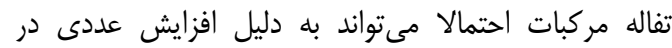

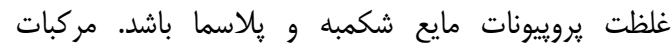

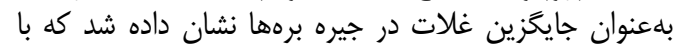

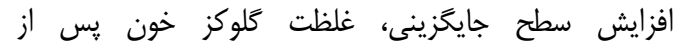

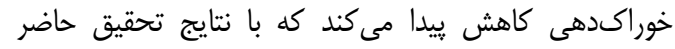

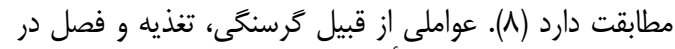

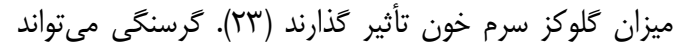

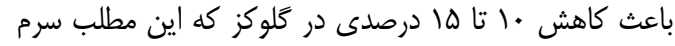

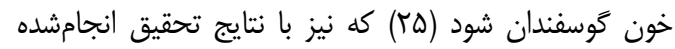

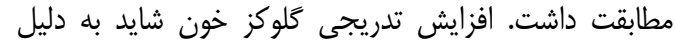

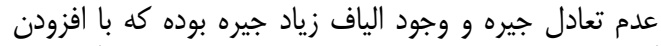

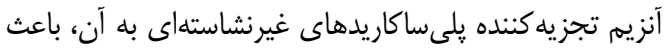

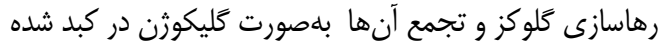

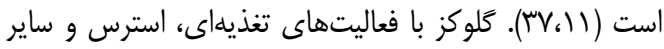

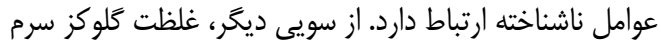

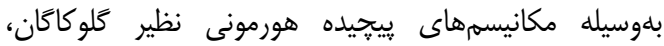
انسولين و ديكر هورمونها نظير كورتيكواستروئيدها، ايينفرين

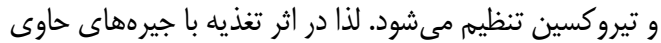

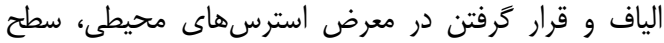

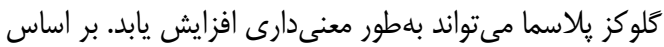

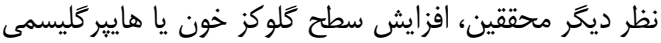


Table 5. Effect of experimental diets on plasma metabolites of Baluchi sheep

جدول ه- اثر جيرههاى آزمايشى بر متابوليتهاى پِاسما كَوسفند بلوخى

\begin{tabular}{|c|c|c|c|c|c|c|}
\hline \multicolumn{6}{|c|}{ جيرههاى آزمايشى * } & \multirow[b]{2}{*}{ فراسنجهها } \\
\hline سطح احتمال & خطاى استانداردميانخين & تيمار & تيمار ب & تيمار r & تيمار & \\
\hline$\cdot / \mu / f f$ & $r / 9 q$. & $r v / V^{e}$ & $\Gamma / \Lambda \Delta$ & $r q / \pi r$ & $r \Delta / r \Delta$ & كلسترول (ميلى گرم در دسى ليتر) \\
\hline .1 .841 & $r / T \Delta L$ & FY/TS & $|r| r \mid$ & $\mu \kappa / l \pi$ & rN/q & اوره (ميلى گرم در دسى ليتر) \\
\hline$\cdot / \pi \mid \cdot 1$ & $r / r q$. & $W / r T$ & $v e / F \Delta$ & $v g / N$ & $\Lambda \cdot / 91$ & كلوكز (ميلى گرم در دسى ليتر) \\
\hline$\cdot / 1 \cdot r)$ & 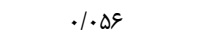 & V/Fr & $\mathrm{V} / \mathrm{r} \Lambda$ & V/ra & V/rT & كل يروتئين پالاسما (كرم در دسى ليتر) \\
\hline ./vas. &.$/ \mathrm{WV}$ & r/Fr & $r / q r$ & $\Gamma / \Delta \Delta$ & $r / \mathcal{L V}$ & آلبومين (كرم در دسى ليتر) \\
\hline . /ADFT & س. & $\cdot / r \checkmark$ & 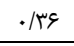 & ع &.$/ \% \Delta$ & بتا هيدروكسى بوتيرات (ميلى كرم در دسى ليتر) \\
\hline
\end{tabular}

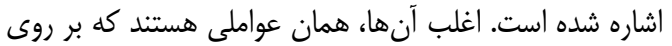

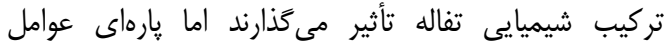

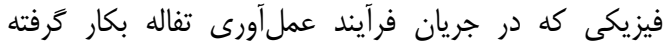

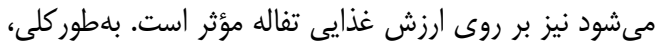

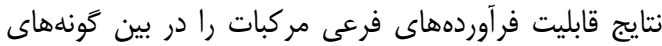

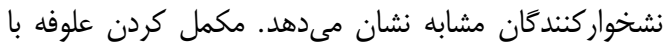

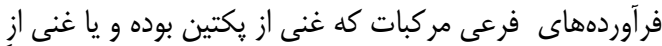

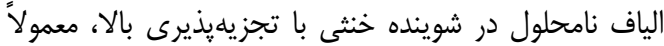

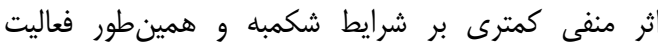

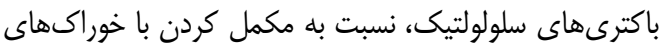

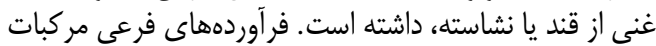

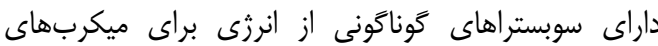

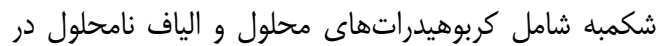

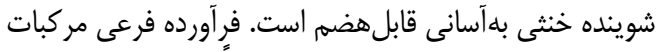

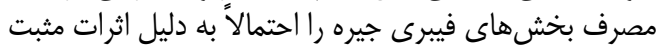

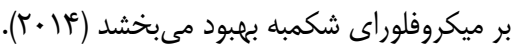

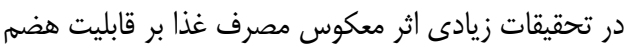

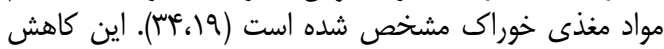

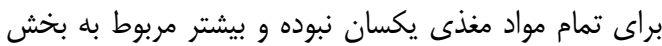

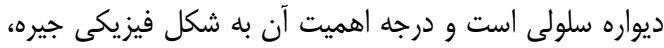

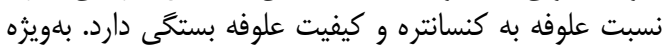

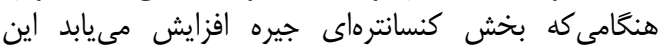

$$
\text { كاهش در قابليت هضم بيشتراست (• (؟). }
$$

\section{قابليت هضم مواد مغذى} نتايج مربوط به اثر جيرههاى آزمايشى بر ضرائ ضرايب قابليت

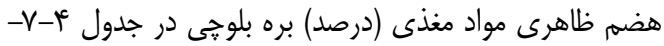

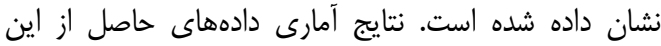

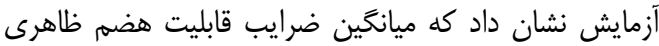

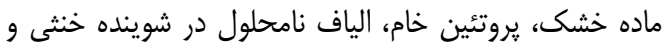

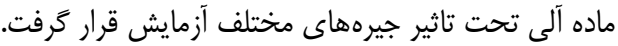

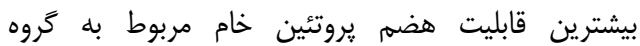

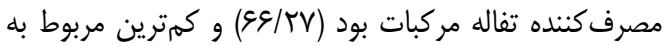

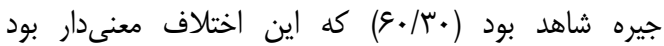

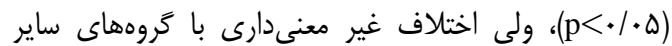
گروهها داشت. در آزمايشى آمرمن و همكاران (ه) ميانخين تركيب مواد

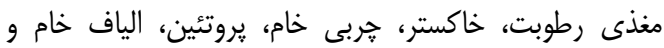

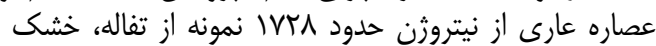

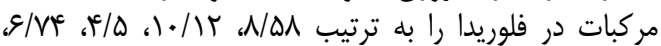

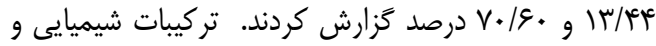

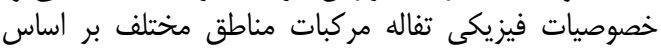

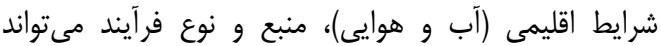

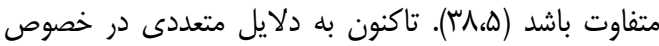

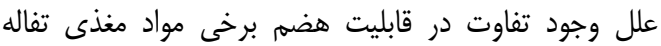

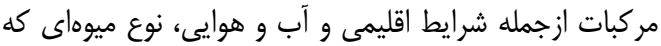

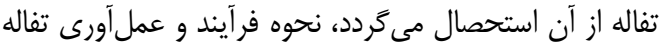

جدول צ- اثر جيرههاى آزمايشى بر ضرايب قابليت هضم ظاهرى مواد مغذى (درصد) برههاى بلوجى Table 6. Effect of experimental diets on the average apparent digestibility coefficient of nutrients of Baluchi lambs (percentages)

\begin{tabular}{|c|c|c|c|c|c|c|}
\hline & \multicolumn{5}{|c|}{ جيرههاى آزمايشى* } & \multirow[b]{2}{*}{ شاخصهاى اندازهيرى شده } \\
\hline سطح احتمال & خطاى استاندارد ميانغين & تيمار f & تيمار r & تيمار r & تيمار 1 & \\
\hline.$/ \cdots 1$ & $1 / \cdot 11$ & sr/rra & $99 / 90^{\mathrm{a}}$ & $99 / 9 \wedge^{\mathrm{a}}$ & $\Delta N / N^{\circ b}$ & ماده خشك \\
\hline 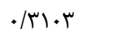 & 1/DQH & $91 / 4 V$ & $\varepsilon \cdot / f \Delta$ & GI/gr & $9 \cdot 111$ & جربى خام \\
\hline.$/ \cdots 1$ & t/IFY & 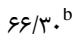 & $9 \Delta / / r^{b}$ & $s q / T V^{b}$ & $\varepsilon \cdot / \mu \cdot{ }^{a}$ & يروتئين خام \\
\hline $.1 \cdots+1$ & 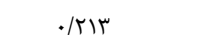 & $R \cdot / 4 m^{a}$ & $M / 1 Q^{\mathrm{a}}$ & $V / r \Lambda^{a}$ & Gr/ATb & ماده آلى \\
\hline$\cdot 1 \cdots+1$ & $r / \cdot r f$ & $\Delta N / 1 \cdot{ }^{b}$ & $q \cdot / r^{b}$ & $98 / 1 V^{b}$ & - ( & الياف نامحلول در شوينده خنثى \\
\hline$\cdot / 4991$ & $1 / 49 V$ & DI/TR & $\Delta \cdot / V \cdot$ & $\Delta T / \mu$. & Q & الياف نامحلول در شوينده اسيدى \\
\hline
\end{tabular}


r. بررسى سطوح متفاوت تفاله خشك جغندرقند و پسماند مر كبات

1. Abedini, A.H., T. Gourchy and S. Zerehdaran. 2012. Effect of replacement of different levels of citrus pellets with barley grain in diet of lambs of Taleshi mass. Animal Production Research, 2: 53-43 (In Persian)

2. Afshar Mazandaran, N. and A. Rajab. 2002. Probiotics and their application in feeding livestock and poultry. Nourbakhsh Publication, (In Persian).

3. Agrahari S., K.C. Pandey and K. Gopal. 2007. Biochemical alteration induced by monocrotophos in the blood plasma of fish, Bloch (Channa punctatus). Pesticide Biochemistry and Physiology, 88(3): 268-272.

4. Allen, M.S. 2000. Effects of diet on short-term regulation of feed intake by lactating dairy cattle. Journal Dairy Science, 83: 1598-1624.

5. Ammerman, C.B., J.F. Easley, L.R. Arrington and F.G. Martin. 1966. Factors affecting the physical and nutrient composition of dried citrus pulp. Proc. Florida State Hor. Soc.

6. AOAC. 1990. Official Methods of Analysis. Vol. I. $15^{\text {th }}$ ed. Association of Official Analytical Chemists, Arlington, VA.

7. Bayat Koohsar, J., R. Valizadeh, A.S. Nasserian, A.S. Tahmasebi and R. Safari. 2010. The effect of barley replacement with citrus dry matter in Holstein dairy cows diet on their performance. Iranian Journal of Animal Science Research. Iranian Journal of Animal Science Research, 2: 148-155 (In Persian).

8. Bhattacharya, A.N. and M. Harb. 1973. Dried citrus pulp as a grain replacement for Awasi lambs. Journal of Animal Science, 36: 1175-1180.

9. Bueno, M.S., J.E. Ferrari, D. Bianchini, F.F. Leinz and C.F.C. Rodrigues. 2002. Effect of replacing corn with dehydrated citrus pulp in diets of growing kids. Small Ruminant Research, 46: 179-185.

10. Caparra P., F. Foti, M. Scerra, M.C. Sinatra and V. Scerra. 2007. Solar-dried citrus pulp as an alternative energy source in lamb diets: Effects on growth and carcass and meat quality. Small Ruminant Research, 68: 303-311.

11. Duncan D.B. 1955. Multiple ranges and Multiple F-test. Biometrics, Aminal nitrogen metabolism: perspectives for integration of microbiology and nutrition for dairy. Journal of dairy science, 90 (E. Suppl.):E1-E16. 11: 1-42.

12. Fazaeli, H. and M. Zahedi Far. 2004. Value of nutrition and application of plant residues in livestock feeding. The first scientific conference on the management of plant remains. Ministry of Agriculture Jihad, 95-96 (In Persian).

13. Fazaeli, H. 2007. The nutritional value of dried pistachio residues along with dried alfalfa in sheep. Proceedings of the 2nd National Congress of Animal Sciences and Aquaculture, National Institute of Animal Science Research. Karaj, 131-137 (In Persian).

14. Fazaeli, H. 2009. Optimum use of agricultural wastes in livestock feed. Fourth National Conference on Agricultural Waste Dissertation, Tarbiat Modares University, 198-201 (In Persian).

15. Fazaeli, H. 2010. Improving livestock nutrition management using full feed technology. Fourth Iranian Congress of Animal Sciences. College of Agricultural of Karaj, 1304-1311 (In Persian).

16. Fegeros, K., G. Zervas, S. Stamouli and E. Apostolaki. 1995. Nutritive value of dried citrus pulp and its effect on milk yield and milk composition of lactating ewes. Journal Dairy Science, 78: 1116-1121.

17. Grant, R.J. 1997. Interactions among forages and non forage fiber sources. Journal Dairy Science, 80: $1438-1446$.

18. Henrique, W., P.R. Leme, D.P.D. Lanna, J.L.V. Coutinho- Filho, R.M. Peres, C.L. Justo, P.A. de Siqueira and G.F. Alleoni. 1998. Replacement of starch for pectin in diet with different concentrate levels Animal performance and carcass characteristics. Revista Brasileira de Zootecnia, 27: 12061211.

19. Khorasani, G.R., E.K. Okine and J.J. Kennelly. 2001. Effects of forage source and amount of concentrate on rumen and intestinal digestion of nutrients in late lactation cows. Journal of dairy science, 84: 1156-1165.

20. Kononoff, P.J., H.A. Lehman and A.J. Heinrichs. 2002. Technical note-A comparison of methods used to measure eating and ruminating activity in confined dairy cattle. Journal of Dairy Science, 85: 1801-1803.

21. Krehbiel C., S. Rust, G. Zhang and S. Gilliland. 2003. Bacterial direct-fed microbials in ruminant diets: Performance response and mode of action. Journal of Animal Science, 81(14_suppl_2): E120.

22. Martin J.L.K. and M.C. Black 1998. Biomarker assessment of the effects of coal-strip mine contamination on channel catfish. Ecotoxicology and Environmental Safety, 41(3): 307-320.

23. McDonald P., R.A. Edwards, J.F.D. Greenhalgh and C.A. Morgan. 1995. Animal Nutrition. Addison Wesley Longman.

24. Mertens, D.R. 1997. Creating a system for meeting the fiber requirements of dairy cows. Journal Dairy Science, 80: 1463-1481.

25. Mojabi A. 1991. Veterinary Clinical Biochemistry. $6^{\mathrm{Ed}}$. Jihad Publications, $372 \mathrm{pp}$. 
26. Nisbet, D.J. and S.A. Martin. 1991. Effect of Saccharomyces cerevisiae culture on lactate utilization by the ruminal bacterium Selenomonas ruminantium Journal of Animal Science, 69: 4628-4633.

27. Nocek J.E. 1997. Bovine acidosis: implications on laminitis. Journal of Dairy Science, 80: 10051028.

28. Nouri S., J. Bayat Koharsar, G.A. Pour Alamdari and F. Ghanbari. 2014. Nutritional value and comparison of different drying methods on the chemical composition, gas production components and digestibility of citrus pulp. Animal and Poultry Research, 3: 69-57 (In Persian).

29. Oni A.O., C.F.I. Onwuka, O.O. Oduguwa, O.S. Onifade, O.M. Arigbede and J.E.N. Olatunji. 2006. Utilization of citrus pulp based diets and enterolobium cyclocarpum foliage by West African Dwarf goats. Journal Animal Veterinary Advances, 5: 814-818.

30. Owens F.N., D.S. Secrist, W.J. Hill and D.R. Gill. 1998. Acidosis in cattle: a review. Journal of Animal Science, 76: 275-286.

31. Parayad A.S. 2004. Effect of Saccharomyces cerevisiae on the digestibility and performance of lamb fattening containing bagasse in sheep. Master's thesis. Razi University of Kermanshah, 95-111 (In Persian).

32. Pirmohammadi, R., O. Hamidi and A. Mohsenpur Azari. 2007. Effects of polyethylene glycol (PEG) addition on composition, degradability digestibility of white grape pomace. Journal Animal Veterinary Advances, 6: 1135-1139.

33. Riddell, J.B., A.J. Gallegos, D.L. Harmon and K.R. Mcleod. 2010. Addition of a Bacillus based probiotic to the diet of pre ruminant calves: influence on growth, health, and blood parameters. Intern. Journal of Applied Research in Veterinary Medicine, 8: 78-85.

34. Rim, J.S., S.R. Lee, Y.S. Cho, E.J. Kim, J.S. Kim and K.H. Jong. 2008. Prediction of dry matter intake in lactating Holstein dairy cows offered high levels of concentrate. Asian-Aust Journal of Animal Science, 21: 677-684.

35. Saedi, H., M. Nikpour Tehrani and A. Morvarid. 1992. Animal Feeds and their Preservation Methods (Principles of animal feeding). Tehran University Press.

36. SAS, Institute. 2003. SAS User's Guide. Version 9.1 ed. SAS Institute Inc., Cary, NC.

37. Schang, M.J. and J.O. Azcona. 1998. Performance of laying hens fed a corn-sunflower diet supplemented with enzymes. In: Lyons T.P., Jacques K.A. (Eds.). Passport to the Year 2000, Biotechnology in the feed Industry. Proceedings of All techs $14^{\text {th }}$ Annual Symposium. Nottingham University Press, Nottingham, UK, pp: 405-410.

38. Scotti, G.G. and A. Lanza. 1973. New knowledge on the amino acid and mineral composition and on digestibility in vitro of dried orange pulp. Alimantazione Animale, 17(2): 47-60.

39. Volanis, M., P. Zoiopoulos and K. Tzerakis. 2004. Effects of feeding ensiled sliced oranges to lactating dairy sheep. Small Ruminant Research, 53: 15-21.

40. Weiss, W.P., D.L. Frobose and M.E. Koch. 1997. Wet tomato pomace ensiled with corn plants for dairy cows. Journal of dairy science, 80: 2896-2900.

41. Welch, J.G. and A.M. Smith. 1971. Effect of beet pulp and citrus pulp on rumination activity. Journal Animal Science, 33: 472-475.

42. White, S.L., G.A. Benson, S.P. Washburn and J.T. Green Jr. 2002. Milk production and economic measures in confinement or pasture systems using seasonally calved Holstein and Jersey cows. Journal of dairy science, 85: 95-104. 


\title{
Investigation of Different Levels of Sugar Beet Pulp and Citrus Residue on Productive Performance of Baluchi Lambs
}

\author{
Moosa Vatandoost ${ }^{1}$, Masood Didarkhah ${ }^{2}$ and Fereshte Jamili $^{3}$ \\ 1- Assistant Professor, Department of Agriculture, Payame Noor University \\ 2- Assistant Professor, Faculty of Agriculture Sarayan, University of Birjand, Birjand, Iran \\ (Corresponding Author: masooddidarkhah@ birjand.ac.ir) \\ 3- Invited Assistant Professor, Faculty of Agriculture Sarayan, University of Birjand, Birjand, Iran \\ Revived: February 17, $2019 \quad$ Accepted: May 19, 2019
}

\begin{abstract}
In livestock nutrition management to increase productivity, finding inexpensive sources of food is a fundamental solution. . The sub-sectors of the agricultural industry in the feeding of ruminant animals make it possible to use them for the production of valuable products such as meat and milk. The purpose of this study was to investigate the effects of using citrus and sugar beet pulp in diet on ruminal performance and fermentation of Baluchi lambs. This research was carried out in a completely randomized design on 40 Baluchi lamb with 4 treatments and 10 lambs pretreatment with an average weight of $30 \mathrm{~kg} \pm 1.5$. The trial period was 75 days. Treatments were: 1 ) control group (base diet $+0 \%$ citrus pulp per head at day 2) base diet +10 $\%$ citrus pulp per head at day 3 ) base diet $+20 \%$ sugar beet pulp dry per head at day 4) basal diet $+10 \%$ citrus pulp $+5 \%$ beet pulp dry per head at day. There was a significant difference between the mean daily weight gain in the whole period between different diets $(\mathrm{P}<0.05)$. The best conversion coefficient was for the group that consumed citrus (6.25) and the control group with the highest conversion factor (7.75), which had a significant difference with other groups $(\mathrm{P}<0.05)$. The results of this study showed that there was no significant difference between the average duration of chewing time, rumination and eating among different diets. According to the results of this study, the use of citrus pulp as a sub.
\end{abstract}

Keywords: Citrus Waste, Rum, Sheep, Sugar Beet 